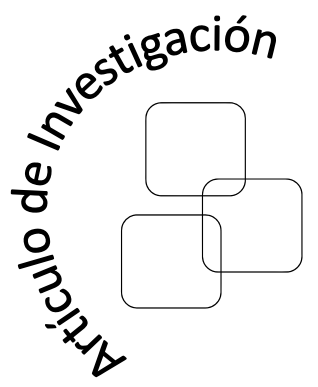

Juan Sebastian Patiño-Callejas Universidad Distrital Francisco José de

Caldas

juansebastianpatinoc@gmail.com

Krisna Yoel Espinosa-Ayala Universidad Distrital Francisco José de

Caldas

joespinosa1018@gmail.com

Juan Carlos Figueroa-García Universidad Distrital Francisco José de

Caldas

jcfigueroag@udistrital.edu.co

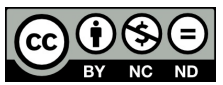

Citación: J. S. Patiño-Callejas, K. J. Espinosa-Ayala y J. C. Figueroa-García. (2015). An approach for solving Goal Programming problems using Interval Type-2 Fuzzy goals. En: Ingeniería, Vol. 20, No. 2, pp. 233-244

(C)Los autores. Titular de los derechos de reproducción: Universidad Distrital Francisco José de Caldas. En línea DOI:

http://dx.doi.org/10.14483/udistrital.jour.reving.2015.2.a04

\title{
An approach for solving Goal Programming problems using Interval Type-2 Fuzzy goals
}

\section{An approach for solving Goal Programming problems using Interval Type-2 Fuzzy goals}

\begin{abstract}
This paper presents a proposal for solving goal problems involving multiple experts opinions and perceptions. In goal programming problems where no statistical data about their goals exist, the use of information coming from experts becomes the last reliable source. This way, we propose an approach to model this kind of goals using Interval Type-2 fuzzy sets, and a simple method for finding an optimal solution based on previous methods that have been proposed for classical fuzzy sets.
\end{abstract}

Key words: Fuzzy linear programming, Interval Type-2 Fuzzy sets, Goal programming.

\section{Resumen}

Este trabajo presenta un acercamiento a la solución de problemas de programación por metas que incluyen la opinión y percepión de múltiples expertos. En problemas de metas que no tienen información estadística adecuada para definir los valores meta, el uso de información proveniente de expertos se convierte en la última fuente confiable de información. Así pues, proponemos una aproximación al modelado de este tipo de problemas utilizando conjuntos difusos de Intervalo Tipo-2, y un método sencillo para encontrar soluciones usando métodos propuestos por otros autores para conjuntos difusos clásicos.

Palabras claves: Programación lineal difusa, Conjuntos difusos Tipo-2 de intervalo, Programación por metas. 


\section{Introduction}

Decision making in practical applications faces multiple issues, including human being interaction and social behavior. Some problems are built over the base of having multiple goals involving multiple experts that try to solve the same problem with different objectives. To solve this kind of situations, goal programming provides a first tool to find crisp solutions.

To handle the problem of having both multiple experts and uncertainty around the exact value of a desired goal, fuzzy sets appear as a useful tool for handling numerical uncertainty coming from experts. Fuzzy goal programming has been proposed by Narasimhan [15], and later developed by Yang [20], Turgay \& Taşkın [18], Li \& Gang [12],Hu, Zhang \& Wang [9], Khalili-Damghani \& Sadi-Nezhad [10], in both theoretical and practical situations.

In decision making, Qin \& Liu [17], Zhang \& Zhang [21], and Chen \& Ting [4] have already used Type-2 fuzzy systems to handle uncertainty coming from multiple experts, so its use in goal programming seems to be feasible.

Based on the model of Narasimhan [15], Yang [20] has proposed a model with fewer variables which obtains the same solution, so what we propose in this paper is to extend their results to a case where multiple experts deal with multiple goals by using Interval Type- 2 fuzzy sets to handle linguistic/numerical uncertainty coming from experts and Linear Programming (LP) methods for handling goal programming.

The paper is organized as follows: Section 1 introduces the main problem. Section 2 presents some basics on fuzzy sets. In Section 3, goal programming LP model is referred. Section 4 presents the Yang [20] proposal for fuzzy goal programming. Section 5 contains the proposal; Section 6 shows an application example; and finally Section 7 presents the concluding remarks of the study.

\section{Basic on Fuzzy sets}

According to Klir \& Yuan [11], the membership function of a fuzzy set $A$ is denoted by $\mu_{A}: X \rightarrow[0,1] . \mathcal{P}$ is the class of all crisp sets, $\mathcal{F}_{1}$ is the class of all fuzzy sets, and $\mathcal{F}_{2}$ is the class of all Type-2 fuzzy sets.

\subsection{Interval Type-2 Fuzzy Sets (IT2FS)}

In general, a Type-2 fuzzy set is simply a function that transforms a set $A$ into the set of fuzzy sets defined over $[0,1]$, this is $\tilde{A}: X \rightarrow \mathbf{F}[0,1]$, where $\mathbf{F}[0,1]$ is also known as the secondary membership function of $\tilde{A}$. An Interval Type-2 Fuzzy set (see Mendel [13]) is an ordered pair $\left\{\left(x, \mu_{\tilde{A}}(x)\right): x \in X\right\}$, where $A$ is a linguistic label $\tilde{A}$ that represents uncertainty about the 
word $A$. Its mathematical definition is:

$$
\begin{gathered}
\tilde{A}=\left(x, \mu_{\tilde{A}}(x)\right): x \in X \\
\tilde{A}=\int_{x \in X} \int_{u \in J_{x}} 1 /(x, u), J_{x} \subseteq[0,1]
\end{gathered}
$$

where $u \in J_{x} \subseteq[0,1]$ is the domain of uncertainty around $A$.

Alternatively, an IT2FS can be fully characterized using two primary membership functions: Lower Membership function (LMF) and Upper Membership Function (UMF) in which are contained all embedded fuzzy sets $A_{e}$ which composes the Footprint of Uncertainty (FOU). Although there are other notations to refer to IT2FSs (see Mendel [14], Türksen [19], and Pagola et al. [16]) who recognize equivalences between Mendel and mathematical standard set notations, we use Mendel notations (see Mendel [13]) due to its interpretability and completeness.

\subsection{Why Fuzzy Sets?}

The main reason for using fuzzy sets is its ability to handle uncertainty coming from human perceptions, which is a common issue in decision making. On the other hand (numerical uncertainty), fuzzy sets can handle imprecision about $X$ which commonly appears when no historical/statistical data is available, so the estimation of the parameters of the problem is based on approximate information coming from the experts of the problem.

\section{Goal programming}

The basic goal programming model proposed by Charnes, Cooper \& Wagner [1], [2] tries to minimize deviations from different goals (desired objectives) through minimizing the absolute deviations $d_{k}$ of the constraints of the problem $A_{k} x$ regarding its desired value (a.k.a goal) $B_{k}$ in the format $\min \left\{D=\sum_{k=1}^{n}\left|A_{k} x-B_{k}\right|\right\}$. This model is equivalent to the following LP model (see Charnes, Cooper \& Wagner [1], [2]):

$$
\begin{gathered}
\min D=\sum_{k=1}^{n} d_{k 1}+d_{k 2} \\
\text { s.t. } \\
A_{k} x+d_{k 1}-d_{k 2}=B_{k}, \\
A_{k}^{\prime} x \leq B_{k}^{\prime} \\
x, d_{k 1}, d_{k 2} \geq 0 ; \forall k \in \mathbb{N},
\end{gathered}
$$

where $B_{k} \in \mathbb{R}$ is the aspiration level, $d_{k 1}, d_{k 2} \in \mathbb{R}$ are negative and positive deviations from the goal $B_{k}, A_{k}$ is the set of $n$ constraints related to goals, $A_{k}^{\prime}$ is a set of crisp constraints of the problem, $B_{k}^{\prime}$ is its set of boundaries, and $x \in \mathbb{R}^{m}$ is the set of decision variables of the problem. A negative deviation quantifies a lack of satisfaction of the desired aspiration level, and a positive deviation quantifies an excess over the desired aspiration level. 


\section{Fuzzy Goal Programming}

Although the first fuzzy goal programming has been proposed by Narasimhan [15], Narasimhan \& Hanna [7], Yang [20] has proposed a model with fewer variables which obtains the same solution of [7], [15]. Yang's proposal starts by defining the membership function of the fuzzy goal $B_{k}$ namely $\mu_{B_{k}}$, as follows:

$$
\mu_{B_{k}}=\left\{\begin{array}{cl}
1-\frac{G_{k}(x)-b_{k}}{b_{k 2}}, & \text { if } b_{k} \leq G_{k}(x) \leq b_{k}+b_{k 2}, \\
1 & \text { if } G_{k}(x)=b_{k} \\
1-\frac{b_{k}-G_{k}(x)}{b_{k 1}}, & \text { if } b_{k}-b_{k 1} \leq G_{k}(x) \leq b_{k}, \\
0 & \text { otherwise, }
\end{array}\right.
$$

where $k \in \mathbb{N}$ denotes the $k_{t h}$ goal, $G_{k}(x)$ is the $k_{t h}$ constraint to be fulfilled, $b_{k} \in \mathbb{R}$ is the aspiration level of the $k_{t h}$ goal, and $d_{k 1}$ and $d_{k 2}$ are the maximum negative and positive deviations from $b_{k}$, respectively. Its LP model is

$$
\begin{gathered}
\min \sum_{k=1}^{n} d_{k 1}+d_{k 2} \\
\text { s.t. } \\
A_{k} x+d_{k 1}-d_{k 2} \cong \tilde{B}_{k}, \\
A_{k}^{\prime} x \leq B_{k}^{\prime} \\
x, d_{k 1}, d_{k 2} \geq 0 ; \forall k \in \mathbb{N},
\end{gathered}
$$

where $\tilde{B}_{k} \in \mathcal{F}_{1}$ the fuzzy aspiration level, $d_{k 1}, d_{k 2} \in \mathbb{R}$ are negative and positive deviations from the goal $b_{k}, A_{k}$ is the set of $n$ constraints related to fuzzy goals, $A_{k}^{\prime}$ is a set of crisp constraints of the problem, $B_{k}^{\prime}$ is its set of boundaries, and $x \in \mathbb{R}^{m}$ is the set of decision variables of the problem.

Finally, the proposal of Yang [20] is based on a simpler LP model in which $G(x) \equiv A_{k} x$, as follows:

$$
\begin{gathered}
\max \lambda \\
\text { s.t. } \\
\lambda \leq 1-\frac{A_{k} x-b_{k}}{b_{k 2}}, \forall k \in \mathbb{N} \\
\lambda \leq 1-\frac{b_{k}-A_{k} x}{b_{k 1}}, \forall k \in \mathbb{N} \\
A_{k}^{\prime} x \leq B_{k}^{\prime} \\
\lambda \in[0,1], x \geq 0 .
\end{gathered}
$$

where $\lambda \in[0,1]$ is the global satisfaction degree of all goals.

This approach uses $\lambda$ as a global variable that represents the satisfaction of every fuzzy goal $\mu_{B_{k}}$. The first constraint represents the satisfaction degree for $b_{k} \leq G_{k}(x) \leq b_{k}+b_{k 2}$ (see Eq. 
(3)), and the second constraint represents the satisfaction degree for $b_{k}-b_{k 1} \leq G_{k}(x) \leq b_{k}$ (see Eq. (3)). As $x$ is a free variable in this model, it operates over $A_{k} x$ and finally moves $\lambda$ to its maximum value.

\section{Goal programming with Interval Type-2 fuzzy aspiration levels}

Disagreement among people who are involved into decision making is a common issue in real scenarios. Some people is pessimistic while others are optimistic about different goals. This leads to have different perceptions coming from different experts, so we handle those perceptions using two functions $L M F$ and $U M F$ which are defined as follows:

$$
\begin{gathered}
U M F \equiv \bar{\mu}_{\tilde{b}_{k}}=\left\{\begin{array}{cl}
1-\frac{G_{k}(x)-b_{k}}{\bar{b}_{k 2},}, & \text { if } b_{k} \leq G_{k}(x) \leq b_{k}+\bar{b}_{k 2}, \\
1-\frac{b_{k}-G_{k}(x)}{\bar{b}_{k 1},}, & \text { if } G_{k}(x)=b_{k}, \\
0 & \text { otherwise, }
\end{array}\right. \\
L M F \equiv \underline{\mu}_{\tilde{b}_{k}}=\left\{\begin{array}{cl}
1-\frac{G_{k}(x)-b_{k}}{\underline{b}_{k 2}}, & \text { if } b_{k} \leq G_{k}(x) \leq b_{k}, \underline{b}_{k 2}, \\
1-\frac{b_{k}-G_{k}(x)}{\underline{b}_{k 1},}, & \text { if } G_{k}(x)=b_{k}-\underline{b}_{k 1} \leq G_{k}(x) \leq b_{k}, \\
0 & \text { otherwise }
\end{array}\right.
\end{gathered}
$$

where $\bar{\mu}_{\tilde{b}_{k}}$ defines the UMF of the $k_{t h}$ goal, and $\underline{\mu}_{\tilde{b}_{k}}$ defines the LMF of the $k_{t h}$ goal. A graphical display of a Interval Type-2 fuzzy goal is shown in Figure 1.

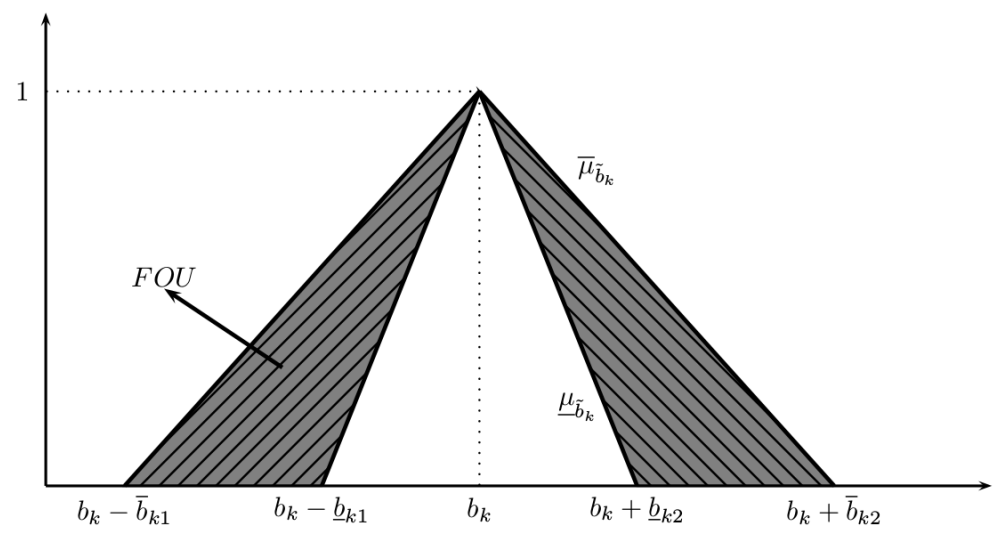

Figure 1. Interval Type-2 Fuzzy goal 
Based on (3) and (5), we extend its results to a Interval Type-2 fuzzy environment. Thus, we define the Interval Type- 2 fuzzy aspiration level as $\tilde{b}_{k}$ which leads to the following LP model:

$$
\begin{gathered}
\min \sum_{k=1}^{n} d_{k 1}+d_{k 2} \\
\text { s.t. } \\
A_{k} x+d_{k 1}-d_{k 2} \approx \tilde{b}_{k}, \\
A_{k}^{\prime} x \leq b_{k}^{\prime} \\
x, d_{k 1}, d_{k 2} \geq 0 ; \forall k \in \mathbb{N},
\end{gathered}
$$

where $\tilde{b}_{k} \in \mathcal{F}_{2}$ is the Interval Type-2 fuzzy aspiration level, $d_{k 1}, d_{k 2} \in \mathbb{R}$ are negative and positive deviations from the goal $B_{k}, A_{k}$ is the set of $n$ constraints, and $x \in \mathbb{R}^{m}$ is the set of decision variables of the problem.

Therefore, we extend the proposal of Yang [20] to a Interval Type-2 fuzzy model using a two-step method that finds two different $\lambda$ values, one for $\bar{\mu}_{\tilde{b}}$ and one for $\underline{\mu}_{\tilde{b}}$. To do so, we have to solve the following two LPs:

$$
\begin{gathered}
\max \bar{\lambda} \\
\text { s.t. } \\
\bar{\lambda} \leq 1-\frac{A_{k} x-b_{k}}{\bar{b}_{k 2}}, \\
\bar{\lambda} \leq 1-\frac{b_{k}-A_{k} x}{\bar{b}_{k 1}}, \\
\bar{\lambda} \in[0,1], x \geq 0 ; \forall k \in \mathbb{N},
\end{gathered}
$$

$$
\begin{gathered}
\max \underline{\lambda} \\
\text { s.t. } \\
\underline{\lambda} \leq 1-\frac{A_{k} x-b_{k}}{\underline{b}_{k 2}}, \\
\underline{\lambda} \leq 1-\frac{b_{k}-A_{k} x}{\underline{b}_{k 1}}, \\
\underline{\lambda} \in[0,1], x \geq 0 ; \forall k \in \mathbb{N}
\end{gathered}
$$

where $\bar{\lambda}$ is the overall upper satisfaction degree of the goals, and $\underline{\lambda}$ is the overall lower satisfaction degree of the goals. $A_{k} x$ is the $k_{t h}$ technological constraint, and $\bar{b}_{k 1}, \underline{b}_{k 1}, \bar{b}_{k 2}, \underline{b}_{k 2}$ are the admissible deviations from $b_{k}$.

Our approach finds two values: $\min \{\underline{\lambda}\}=\underline{\lambda}^{*}$ and $\max \{\bar{\lambda}\}=\bar{\lambda}^{*}$ that represent pesimistic and optimistic perceptions about $b_{k}$, and also compose the interval $\left[\underline{\lambda}^{*}, \bar{\lambda}^{*}\right]=\left\{\lambda^{*} \in\right.$ $\left.[0,1] \mid \underline{\lambda}^{*} \leqslant \lambda^{*} \leqslant \bar{\lambda}^{*}\right\}$ of satisfaction of all experts. 


\section{Experimentation and Results}

\subsection{Interval Type-2 Fuzzy Goals}

As application example we use the proposed by Narasimhan [15] and extended by Chen \& Tsai [3] which is composed by three fuzzy goals, as shown as follows:

$$
\begin{gathered}
G_{1}: 80 x_{1}+40 x_{2} \cong 630, \\
G_{2}: x_{1} \cong 7, \\
G_{3}: x_{2} \cong 4,
\end{gathered}
$$

where $x_{1}$ and $x_{2}$ are the manufacturing quantities of two products which regard to three goals: $G_{1}$ is a profitability goal, and $G_{2}, G_{3}$ are the expected selling quantities per product. The maximum deviations from $G_{k}=\{630,7,4\}$ are symmetrically handled where $b_{k 1}=b_{k 2}=$ $\{10,2,2\}$.

We use those values as the LMF of an extended problem e.g $\underline{b}_{k 1}=\underline{b}_{k 2}=\{10,2,2\}$, and the UMF is defined using $\bar{b}_{k 1}=\bar{b}_{k 2}=\{15,3,3\}$. Now, their LPs are based on Eqs. (9) and (10):

$$
\begin{gathered}
\max \bar{\lambda} \\
\text { s.t. } \\
\bar{\lambda} \leq-\frac{16}{3} x_{1}-\frac{8}{3} x_{2}+43, \\
\bar{\lambda} \leq \frac{16}{3} x_{1}+\frac{8}{3} x_{2}-41, \\
\bar{\lambda} \leq-\frac{x_{1}}{3}+\frac{10}{3}, \\
\bar{\lambda} \leq \frac{x_{1}}{3}-\frac{4}{3}, \\
\bar{\lambda} \leq-\frac{x_{2}}{3}+\frac{7}{3}, \\
\bar{\lambda} \leq \frac{x_{2}}{3}-\frac{1}{3}, \\
\bar{\lambda}, x_{1}, x_{2} \geq 0 \\
\max \underline{\lambda} \\
s . t . \\
\underline{\lambda} \leq-8 x_{1}-4 x_{2}+64, \\
\underline{\lambda} \leq 8 x_{1}+4 x_{2}-62, \\
\underline{\lambda} \leq-0.5 x_{1}+4.5, \\
\underline{\lambda} \leq 0.5 x_{1}-2.5, \\
\underline{\lambda} \leq-0.5 x_{2}+3, \\
\underline{\lambda} \leq 0.5 x_{2}-1 \\
\underline{\lambda}, x_{1}, x_{2} \geq 0 \\
\end{gathered}
$$


The solution of the model (12) is $\bar{\lambda}^{*}=0.76$ reached by $\left(x_{1}, x_{2}\right)=(6.28,3.28)$ and the solution of (13) is $\underline{\lambda}^{*}=0.64$ with $\left(x_{1}, x_{2}\right)=(6.28,3.28)$. A graphical description of the results can be seen in Figures 3, 4 and 5 respectively (see Appendix 1). The optimal value of the goals 1,2 and 3 are 633.6, 6.28 and 3.28 respectively for both $\bar{\lambda}^{*}$ and $\underline{\lambda}^{*}$.

Both models reach the same values of the decision variables $x_{1}$ and $x_{2}$ which is a logical solution since all deviations are defined as $L_{1}$ distances using $\bar{\lambda}$ and $\underline{\lambda}$ as linear functions of $d_{k 1}$ and $d_{k 2}$. The optimal values $\bar{\lambda}^{*}=0.76$ and $\underline{\lambda}^{*}=0.64$ are global satisfaction degrees, which means that all three goals are satisfied at the same level.

\subsection{Type-1 embedded goals}

Now, we solve another example to illustrate how an embedded Type-1 fuzzy set $\left(B_{e}\right)$ into $\operatorname{FOU}\left(\tilde{b}_{k}\right)$ works. To do so, we have selected the deviations for goals 1,2 and 3 as $12.5,2.5,2.5$ which corresponds to the middle point of the support of $F O U\left(\tilde{b}_{k}\right)$ as shown in Figure 2:

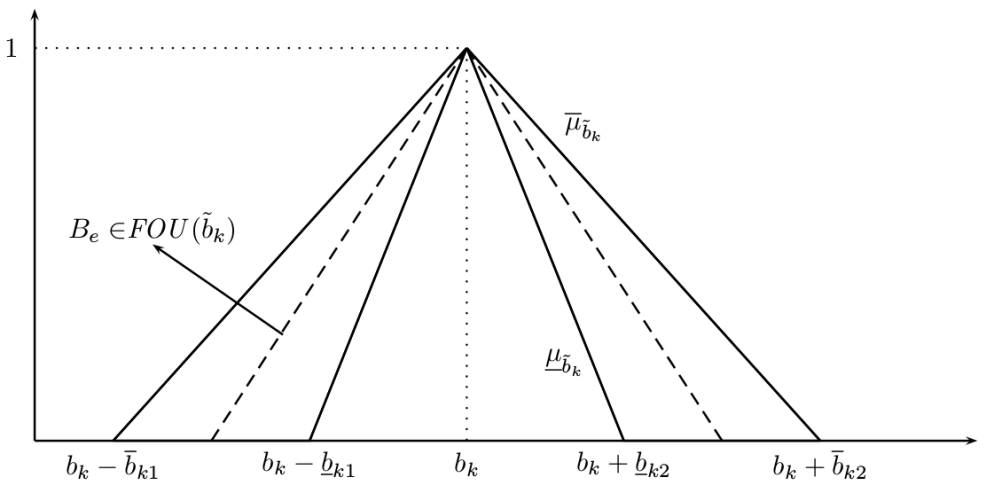

Figure 2. Type-1 embedded set $B_{e}$

The LP formulation based on Eq. (5) is:

$$
\begin{gathered}
\max \lambda \\
\text { s.t. } \\
\lambda \leq-6.4 x_{1}-3.2 x_{2}+51.4 \\
\lambda \leq 6.4 x_{1}+3.2 x_{2}-49.4 \\
\lambda \leq-0.4 x_{1}+3.8 \\
\lambda \leq 0.4 x_{1}-1.8 \\
\lambda \leq-0.4 x_{2}+2.6 \\
\lambda \leq 0.4 x_{2}-0.6 \\
\lambda, x_{1}, x_{2} \geq 0
\end{gathered}
$$

The crisp solution to the problem (14) given Type- 1 fuzzy goals is $\lambda^{*}=0.712$ which fits into the obtained range $\left[\underline{\lambda}^{*}=0.64, \bar{\lambda}^{*}=0.76\right]$ obtained through (9) and (10). Also note that any 
$B_{e} \in \mathcal{F}_{1}$ embedded into $\operatorname{supp}\left(\tilde{b}_{k}\right)$ leads to an optimal satisfaction degree $\lambda^{*}$ that fits into the range $[\underline{\lambda}, \bar{\lambda}]$ as described in the Appendix 2. By continuity of LP models (see Dantzig [5], Hladík [8], and Fiedler et al [6]), if $\tilde{b}_{k} \in \mathcal{F}_{2}$ is continuous then its UMF leads to an optimal solution namely $\bar{\lambda}^{*}$ and every $B_{e} \in F O U\left(\tilde{b}_{k}\right)$ leads to an optimal satisfaction degree, namely $\lambda_{e}^{*} \in[\underline{\lambda}, \bar{\lambda}]$.

\section{Concluding Remarks}

We have presented and solved an extension of the fuzzy goal programming basic model proposed by Narasimhan [15], Yang [20], and Chen \& Tsai [3] to a Interval Type-2 fuzzy environment, which includes linguistic uncertainty and numerical imprecision coming from multiple experts opinions and perceptions.

Our approach gives the model flexibility to find other kind of solutions in cases where the system has no the ability to fulfill all goals. As higher $\lambda^{*}$ as closer to the goal the model is. $\bar{\lambda}$ and $\underline{\lambda}$ describe overall optimistic and pessimistic satisfaction degrees regarding different experts of the system.

There is a relationship among $\lambda^{*}, d_{k 1}$ and $d_{k 2}$ since as wider $\tilde{b}_{k}$ as higher $\lambda^{*}$ is, which means higher satisfaction values. In the first example if $d_{k 1}$ and $d_{k 2}$ are increased in a 50\% then $\lambda^{*}$ is increased only in $12 \%$, and if $d_{k 1}$ and $d_{k 2}$ are decreased in a 50\% then $\alpha^{*}$ is decreased in $36 \%$ (LP formulations for $50 \%$ decreased $d_{k 1}, d_{k 2}, \bar{\lambda}$ and $\underline{\lambda}$ are shown in Appendix 2). Finally, all experts are satisfied into the range $[\underline{\lambda}=0.64, \bar{\lambda}=0.76]$.

The second example shows an embedded set $A_{e}$ into $F O U\left(\tilde{b}_{k}\right)$ whose optimal satisfaction degree $\lambda^{*}$ fits into the range $[\underline{\lambda}, \bar{\lambda}]$ as described in the Appendix 2. This helps decision making when having multiple experts and helps to see how different selections of $A_{e}$ affect the problem.

\section{References}

[1] A. Charnes and W. W. Cooper. Management models and industrial applications of linear programming, vol. i, 1961.

[2] Abraham Charnes and William Wagner Cooper. Goal programming and multiple objective optimizations: Part 1. European Journal of Operational Research, 1(1):39-54, 1977.

[3] Liang-Hsuan Chen and Feng-Chou Tsai. Fuzzy goal programming with different importance and priorities. European Journal of Operational Research, 133(3):548-556, 2001.

[4] Ting-Yu Chen. An electre-based outranking method for multiple criteria group decision making using interval type-2 fuzzy sets. Information Sciences, 263:1-21, 2014.

[5] George Dantzig. Linear Programming and Extensions. Princeton University Press, 1963.

[6] M. Fiedler, J. Nedoma, J. Ramík, J. Rohn, and K. Zimmermann. Linear Optimization Problems with Inexact Data. Springer-Verlag, 2006.

[7] Edward L Hannan. On fuzzy goal programming*. Decision Sciences, 12(3):522-531, 1981. 
[8] Milan Hladík. Weak and strong solvability of interval linear systems of equations and inequalities. Linear Algebra and its Applications, 438(11):4156-4165, 2013.

[9] Chaofang $\mathrm{Hu}$, Shaokang Zhang, and $\mathrm{Na}$ Wang. Enhanced interactive satisficing method via alternative tolerance for fuzzy goal programming with progressive preference. Applied Mathematical Modelling, 38(19):46734685, 2014.

[10] Kaveh Khalili-Damghani, Soheil Sadi-Nezhad, and Madjid Tavana. Solving multi-period project selection problems with fuzzy goal programming based on topsis and a fuzzy preference relation. Information Sciences, 252:42-61, 2013.

[11] George Klir and Bo Yuan. Fuzzy sets and fuzzy logic, volume 4. Prentice Hall New Jersey, 1995.

[12] Gang Li. Fuzzy goal programming-a parametric approach. Information Sciences, 195:287-295, 2012.

[13] Jerry M Mendel. Uncertain rule-based fuzzy logic system: introduction and new directions. Prentice Hall PTR, 2001.

[14] Jerry M. Mendel. On centroid computations for Type-2 fuzzy sets. Appl. Comput. Math., 10(1):88-96, 2011.

[15] Ram Narasimhan. Goal programming in a fuzzy environment. Decision sciences, 11(2):325-336, 1980.

[16] M. Pagola, C. Lopez-Molina, J. Fernandez, E. Barrenechea, and H. Bustince. Interval type-2 fuzzy sets constructed from several membership functions. application to the fuzzy thresholding algorithm. IEEE Transactions on Fuzzy Systems, 21(2):230-244, 2013.

[17] Jindong Qin and Xinwang Liu. Multi-attribute group decision making using combined ranking value under interval type-2 fuzzy environment. Information Sciences, 297:293-315, 2015.

[18] Turgay Safiye and Taşkın Harun. Fuzzy goal programming for health-care organization. Comp. \& Ind. Eng., 2014.

[19] I.B. Türksen. Interval valued fuzzy sets based on normal forms. Fuzzy Sets and Systems, 20(2):191-210, 1986

[20] Taeyong Yang, James P Ignizio, and Hyun-Joon Kim. Fuzzy programming with nonlinear membership functions: piecewise linear approximation. Fuzzy sets and systems, 41(1):39-53, 1991.

[21] Zhiming Zhang and Shouhua Zhang. A novel approach to multi attribute group decision making based on trapezoidal interval type-2 fuzzy soft sets. Applied Mathematical Modelling, 37(7):4948-4971, 2013. 


\section{A Appendix 1}

This appendix contains the results of the optimization process for the first Interval Type-2 fuzzy goals example.

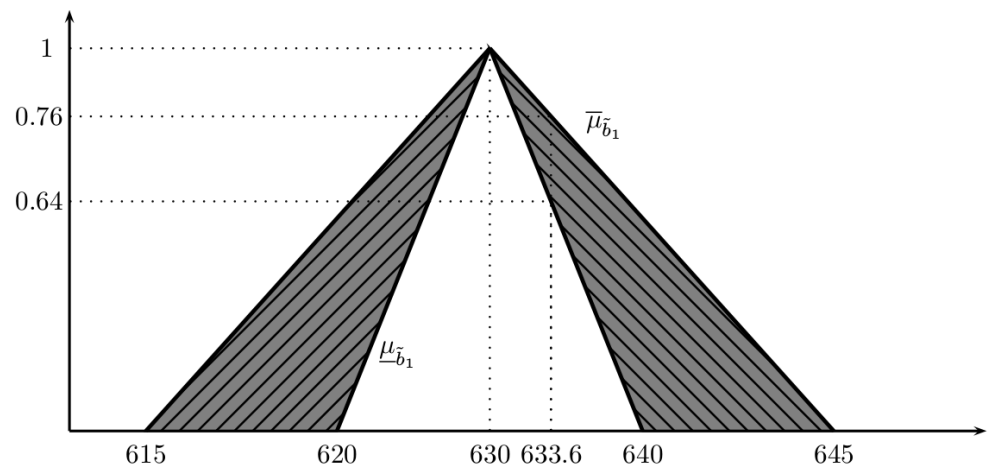

Figure 3. Interval Type-2 Fuzzy Goal 1

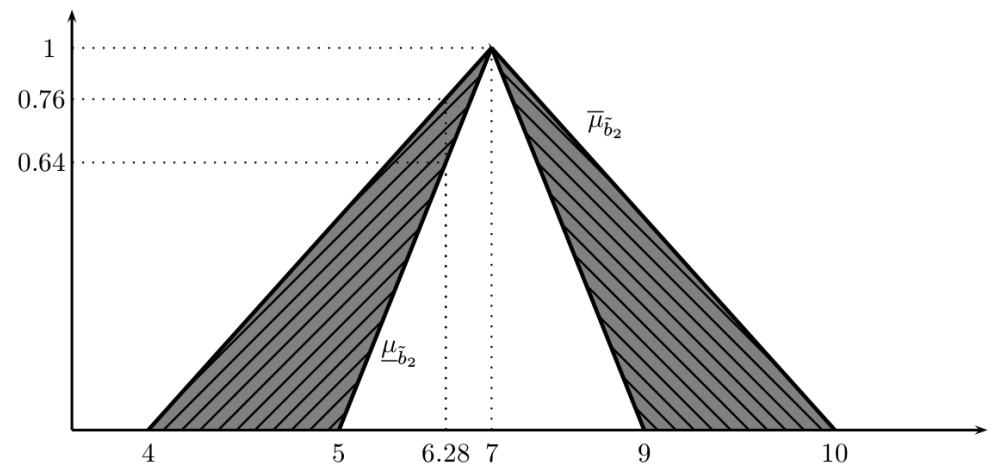

Figure 4. Interval Type-2 Fuzzy Goal 2

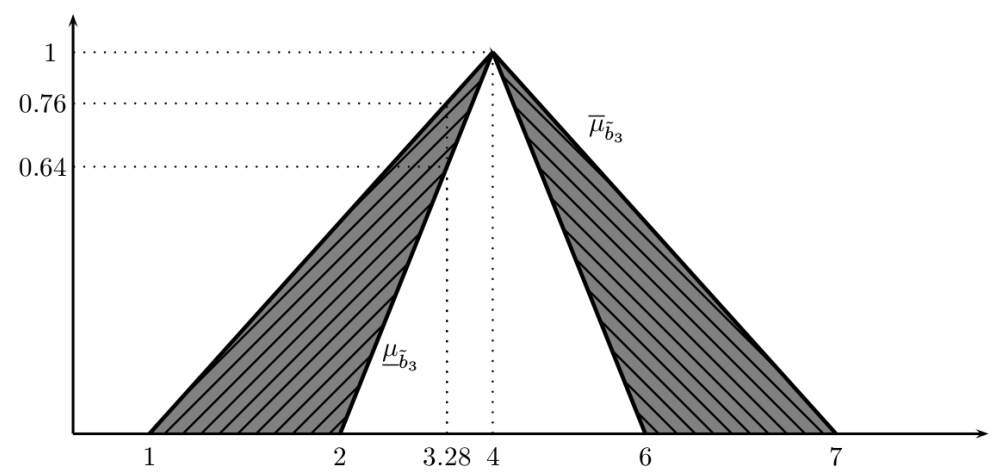

Figure 5. Interval Type-2 Fuzzy Goal 3 


\section{B Appendix 2}

This appendix shows the model of the Type- 1 example shown in Section 6 where $\underline{b}_{k 1}=\underline{b}_{k 2}=$ $\{5,1,1\}$ and $\bar{b}_{k 1}=\bar{b}_{k 2}=\{10,2,2\}$ :

$$
\begin{gathered}
\max \underline{\lambda} \\
\text { s.t. } \\
\underline{\lambda} \leq-16 x_{1}-8 x_{2}+127, \\
\underline{\lambda} \leq 16 x_{1}+8 x_{2}-125, \\
\underline{\lambda} \leq-x_{1}+8, \\
\underline{\lambda} \leq x_{1}-6, \\
\underline{\lambda} \leq-x_{2}+5, \\
\underline{\lambda} \leq x_{2}-3, \\
\underline{\lambda}, x_{1}, x_{2} \geq 0 \\
\max \bar{\lambda} \\
s . t . \\
\bar{\lambda} \leq-8 x_{1}-4 x_{2}+64, \\
\bar{\lambda} \leq 8 x_{1}+4 x_{2}-62, \\
\bar{\lambda} \leq-0.5 x_{1}+4.5, \\
\bar{\lambda} \leq 0.5 x_{1}-2.5, \\
\bar{\lambda} \leq-0.5 x_{2}+3, \\
\bar{\lambda} \leq 0.5 x_{2}-1, \\
\bar{\lambda}, x_{1}, x_{2} \geq 0
\end{gathered}
$$

\section{Juan Sebastian Patiño-Callejas}

He performs Industrial Engineering studies at the Universidad Distrital Francisco José de Caldas - Bogotá, Colombia. e-mail: juansebastianpatinoc@gmail.com

\section{Krisna Yoel Espinosa-Ayala}

He performs Industrial Engineering studies at the Universidad Distrital Francisco José de Caldas - Bogotá, Colombia. e-mail: joespinosa1018@gmail.com

\section{Juan Carlos Figueroa-García}

He is an Assistant Professor at the Engineering Faculty of the Universidad Distrital Francisco José de Caldas - Bogotá, Colombia. He obtained his bachelor degree on Industrial Engineering at the same university in 2002, a Master degree on Industrial Engineering at the same university in 2010, and a Ph.D. degree on Industry and Organizations at the Universidad Nacional de Colombia in 2014. His main interests are: fuzzy sets, fuzzy optimization, time series analysis and evolutionary optimization. e-mail: jcfigueroag@udistrital.edu.co 